\title{
Listen to me: Why narrative medicine matters in healthcare
}

\author{
Susana Teixeira Magalhães \\ University Fernando Pessoa, \\ Institute of Bioethics, Portuguese Catholic University, \\ R. Diogo de Botelho, 1327,4169-005 Porto, Portugal
}

\begin{abstract}
In contemporary societies, the relationship between patients and health professionals is undergoing substantial changes, together with the growing recognition of the former as active participants in deliberation and decision making on the management of their condition. Accordingly, adequate knowledge of disease/illness is necessary to set up a dialogue between patients/citizens and experts/professionals. In the health field, narratives constitute the axial point between distinct but complementary realities, emphasizing that the physiological meaning of disease falls short of individual and social translation. These renewed alignments of experiential and biomedical knowledge determine not only physicians' involvement, but they also require reconsidering the training of health and humanities students and their role in the care of patients. Moreover, hospital managers, journalists, civil organizations, government and patients' associations are elicited to participate in the outline of new borders and new landscapes in healthcare. The intra-subjective and inter-subjective dimensions of Medicine include not only the patient-doctor encounter, but also all the other members of the therapeutic relationship - relatives, other health professionals, community -, including teams and organizations. Listening to all these participants in healthcare is mandatory in order to face the ethical, scientific and technological challenges of a fast changing world. Narrative Medicine provides the tools and the skills to promote listening, interpretation, representation and affiliation, standing out as the interdisciplinary field of knowledge that can build the bridge to the future of a more humane healthcare. ( 232 words)
\end{abstract}

Keywords: Narrative Medicine; Interdisplinary Knowledge; Healthcare; Narrative Skills

\section{INTRODUCTION}

In contemporary societies, the relationship between patients and health professionals is undergoing substantial changes, together with the growing recognition of the former as active participants in deliberation and decision making on the management of their condition. Accordingly, adequate knowledge of disease/illness is necessary to set up a dialogue between patients/citizens and experts/professionals. In healthcare, narratives constitute the axial point between distinct but complementary realities, emphasizing that the physiological meaning of disease falls short of individual and social translation. These renewed alignments of experiential and biomedical knowledge determine not only physicians' involvement, but they also require reconsidering the training of health and humanities students and their role in the care of patients. Moreover, hospital managers, journalists, civil organizations, government and patients' associations are elicited to participate in the outline of new borders and new landscapes in healthcare.

Narrative medicine, the main contributor to the changing scenery briefly described above, is now at a crossroad: having made the diagnosis of the faulty elements within the territory of healthcare, it is high time to build the bridge from prescription to action. So, at present the issue that needs to be addressed is how narrative medicine can be implemented in clinical 
practice. The inter-subjective dimension of therapeutic relationships and the relevance of narrative knowledge in healthcare recognized by Narrative Medicine demand from the health professionals the development of narrative skills that enable them to recognize suffering, interpret and be moved to act on the stories of illness. Although the focus has been on patient care, the role of narrative in medicine is perceived as much wider and deeper, reaching not only the quality of patient care but also the three Rs of good medical practice: Relationship, Recognition of one's own and of others' vulnerability, Reflection (or, we shall say, Responsibility, Respect, Reconciliation)

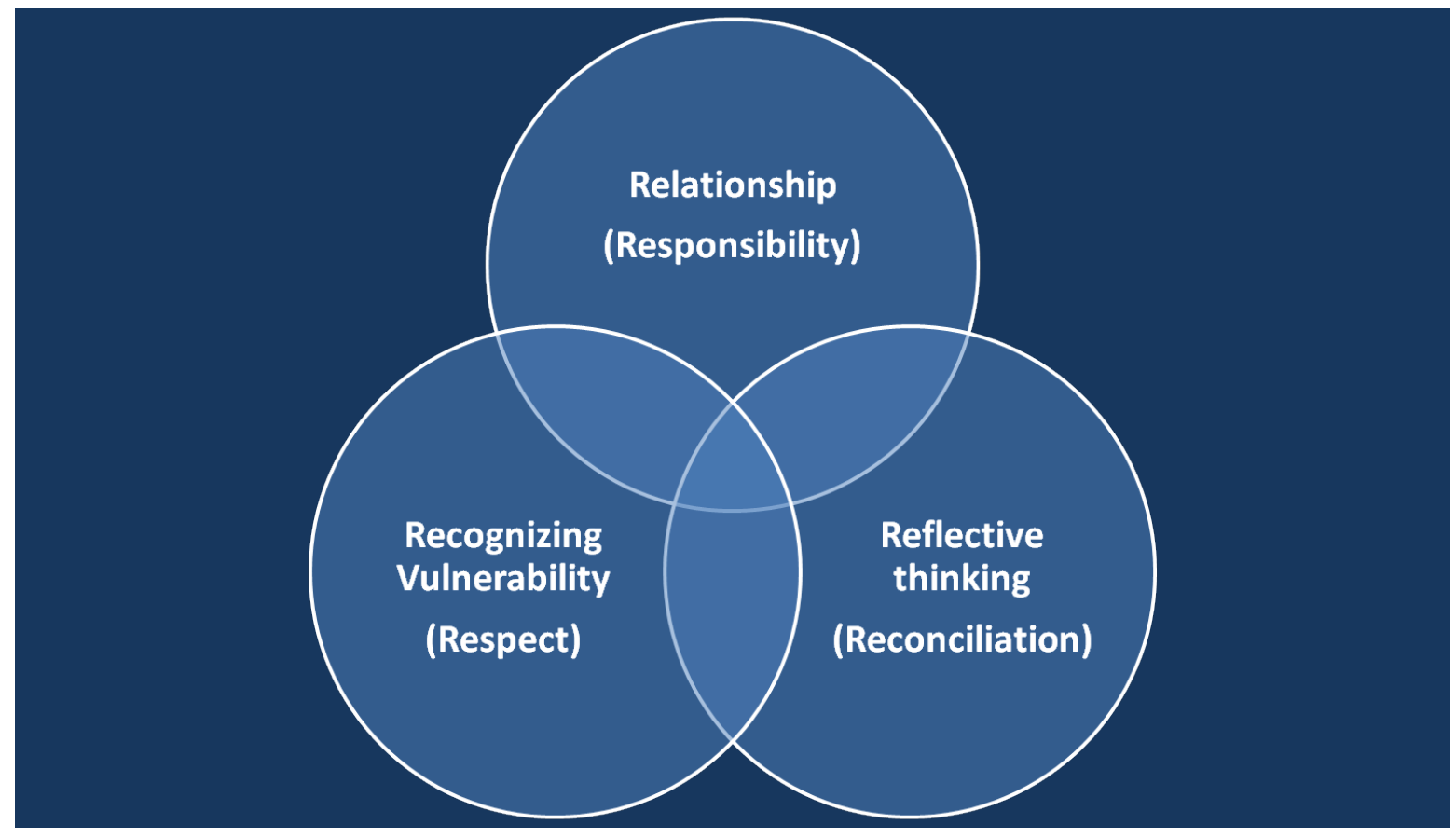

\section{Narrative-based Medicine: Searching for personhood amidst patienthood and professionalism}

The principles mentioned above provide the basis for integrating the meaning of the disease described in scientific language (disease) with the meaning of the disease for the person who lives it (illness) and also with the way society interprets it (sickness). The need to provide thickness to care led to the emergence of Narrative Medicine (NM), defined by Rita Charon as medicine practised with narrative competence to recognize suffering, to interpret and be sensitized by the history of the disease and by the vulnerability of patients, team colleagues and ourselves ${ }^{1}$. This is an interdisciplinary area, grounded in Humanities and Health Sciences, that stemmed from the to the so-called linguistic turn in the early $20^{\text {th }}$ century, which focused on language as instrumental to the perception of behavior or events. The visibility of the shaping role of language at doctor-patient encounter fundaments the refusal of the reductionism underpinning current standard medical practice, which is too much dominated by diagnostic tests, especially imaging techniques.

By shifting the focus to context and to the narratives of each person inhabiting it, Narrative Medicine stands out "as a practice and an intellectual stance which enables physicians to look beyond the biological mechanisms at the centre of conventional approaches to medical

${ }^{1}$ Rita Charon. 2001. "Narrative Medicine: Form, Function, and Ethics". Ann Intern Med.;134, pp. 83-87. doi:10.7326/0003-4819-134-1-200101020-00024 
practice, towards domains of thought and ways of telling" that highlight "language and representation, emotions and relationships illuminating health care practice." ${ }^{2}$

In health care, standardization, the focus on diagnostic technology, the search for objectivity translated into guidelines and check-lists, with no room for the intersubjectivity of human interactions, led to the search for another paradigm, capable of integrating the experience of those who suffer and care with accurate, objective and measurable scientific knowledge:

"Narrative Medicine medicine emerged to challenge a reductionist, fragmented medicine that holds little regard for the singular aspects of a patient's life and to protest the social injustice of a global healhcare system that countenances tremendous healh disparities and discriminatory policies and practices." 3

By integrating all health professionals and not just physicians, the main goal of NM is to preserve human interaction as the means par excellence of therapeutic care. The aim is to set up a dialogue between the best of evidence-based medicine (defined as the conscious and judicious use of the best and most up-to-date scientific data from clinical research, taking into account the specificity of each patient "4), and the best of narrative-based medicine, underlining the importance of context, of the unique nature of both those who need to be cared for and the caregivers. The construction of this bridge requires rethinking concepts, gestures, attitudes and practices, reflecting on the impact of labels and invisible stories; it implies getting out of the comfort zone, recognizing that scientific and technological knowledge often fails to respond to suffering. Medical humanities, person-centered care and NM are not the same, since the latter is broader and deeper:

- NM does not only think about the narratives that unfold in healthcare, rather it reflects essentially with these narratives, allowing them to act and to be effective throughout time and space.

- As relationship-based Medicine, NM can also help to identify and to understand the factors that promote or prevent true care centered on the person. By reflecting with and not only about narratives, NM promotes the sharing of experiences and ethical dilemmas among members of the same team and of different teams, including professionals from diverse backgrounds.

- Interestingly, NM can also promote the integration of evidence into the world of practice. According to Gabbay and le May, in their ethnographic study of knowledge management in primary care ${ }^{5}$, the power of ingrained beliefs and tacit guidelines - the so called mindlines -- is an obstacle to bridging the gap between evidence and practice. In order to articulate these two fields - research and practice --, one should resort to narrative strategies that allow for patients to tell their stories and for health professionals to face the emotions that may predispose them to low-value care. ${ }^{6}$

- NM promotes ethical sensitivity, reducing moral residues; it illuminates recurrent metaphors; it provides input for the imaginary representation of the disease experience.

\footnotetext{
${ }^{2}$ Hurtwitz B. Narrative (in) Medicine. In: Spinozzi P, Hurtwitz B, (Eds). 2011. Discourses and Narrations in the Biosciences. Göttingen: Vandenhoeck \& Ruprecht Unipress, p.73.

3 Rita Charon, Sayantani DasGupta, Nellie Hermann, Craig Irvine, Eric R. Marcus, Edgar Rivera Colsn, Danielle Spencer, and Maura Spiegel. 2016. The Principles and Practice of Narrative Medicine . London: O.U.P., p. 1.

${ }^{4}$ Sackett DL, Rosenberg WM, Gray JA, Haynes RB, Richardson WS. 1996. "Evidence Based Medicine: what it is and what it isn't". Br Med J., 312, pp. 71-2.

5 Gabbay J and May AI. 2004. "Evidence based guidelines or collectively constructed "mindlines?" ethnographic study of knowledge management in primary care”. BMJ, 329 (7473), p. 1013.

6 Ian A Scott, Jason Soon, Adam G Elshaug and Robyn Lindner. 2017. "Countering cognitive biases in minimising low value care”. The Medical Journal of Australia. 206 (9), pp. 407-411. doi: 10.5694/mja16.00999.
} 
The pedagogical instruments proposed by Charon are mainly:

- reading fictional texts, following the close reading method as a training methodology that provides the trainees with room and time to reflect upon their own practices, attitudes and beliefs;

- reflective writing, as a good tool for raising awareness on obstacles, problems and solutions faced by health professionals, patients and their families, managers and decision makers;

- parallel chart, i.e. the register of medical records that can integrate and connecte biography with biology. Charon postulates the hypothesis that the practice of reading and writing promotes the capacities of attention, representation and affiliation that translate into relationship-centred healthcare, in which it is possible to keep personhood together with patienthood and professionalism. Other NM approaches have been postulated, notably by Maria Giulia Marini, whose research team has contributed to the dialogue between different health care actors through the collection and the semantic analysis of real narratives. As a practice tool, the parallel chart should be integrated into the electronic medical record on a regular basis and not as an exceptional resource. This is one of the challenges that NM is facing nowadays, together with the need to disclose the importance of technology in a narrative-evidence-based healthcare world.

\section{Listen to me: Narrative-medicine oriented listening}

In contrast with the traditional role of Medical Humanities, mainly conceived as input that can enrich the training of health professionals, without actually inhabiting neither their practice nor the health system organization, NM aims at integrating this multidisciplinary field as an effective response to illness and care. By developing narrative competences not only among physicians but also among all health professionals, narrative medicine differentiates itself form patient-centred care, because its main focus is not to collect more data about the patient, but to set up relationships within the healthcare environment that allow for the integration of personal data in patients care, as well as in team work and in individual practice. It is not only about gathering more data; it is about questioning the nature of knowledge and how knowledge gains legitimacy. ${ }^{7}$ Therefore, we would say, that at the core of NM is intersubjectivity and this relational approach has got an impact on the quality of communication and care. Listening is crucial to setting up relation-based medicine, but it will always fall short of its target if it is merely patient-centred, i.e., if it only entails attention and details monitoring. In order to be actually narrative medicine-oriented listening it should imply co-creation of knowledge, which means that attention should be paid not only to the details shared by patients, but to their interpretation by patients themselves, as well as to how the interaction between patient-physician/other health professionals can shape the proper act of communication. Instead of unearthing data from patients, narrative medicine-oriented physicians should aim at unraveling the meaning of the facts: "Symptoms, accordingly, should be not merely documented but situated within the stories patients weave to make sense of their lives. How these patients interpret and organize their lives, including their illnesses, is the focus of attention."8

\footnotetext{
7 John W Murphy; Jung Min Choi; Martin Cadeiras.. 2016. "The Role of Clinical Records in Narrative Medicine: A Discourse of Message". The Permanente Journal, Spring;20(2), pp.103-108. http://dx.doi.org/10.7812/TPP/15101.

8 Ibid, p. 103.
} 


\section{Relocating the person: Reconceptualization as reconciliation}

The relational approach of narrative medicine requires two main tasks from health professionals: relocating personhood within patienthood and professionalism by means of acknowledging the subjective elements at play at the clinical encounter; and reconceptualizing terms and concepts as means to reconciliation with their own selves and with all those who take part in health care. The skills needed to give depth to the clinical encounter can be found in the writing and reading of literary texts; but the input required to find this depth, to make a two-dimension world change into a third-dimension one, needs more than literary readings and writings, requiring also real narratives from patients, their relatives and health professionals.

These real narratives shed light on the so-called epistemic injustice, which comprises two forms, according to Miranda Fricker': testimonial injustice and hermenutic injustice. The former takes place when "prejudice causes a hearer to give a deflated level of credibility to a speakers' word", whereas the latter occurs when there is a gap in collective interpretative resources that puts someone at an unfair disadvantage when it comes to making sense of their social experience. We believe that these two forms of epistemic injustice can only be tackled if health professionals have time and space to reflect upon the subjective elements that play a role in the therapeutic relationship, including the terms and the concepts they use within this context. Moreover, we also believe that by disclosing subjectivity as part of their professional performance and not as something negative that they should shun, health professionals will be more able to integrate error as part of their life, learning from it and avoiding moral residues that block their own narratives.

\section{LABELS AND ERRORS}

Acknowledging subjectivity means being able to face prejudice and how it may affect misdiagnosis and patients compliance. If physicians and other health professionals can actually shape what patients tell, by their posture, tone of voice, the words they say and those that remain unsaid or distorted, then communication strategies fall short of their target if they are just a list of what to say and how to say it. Training in communication should also include self-reflection on the impact of labelling on the quality of care. A patient labelled as noncompliant also has a narrative to tell. Only when this narrative is listened to and integrated in the plan of care, can the person who is ill stand out from the restricted territory of patiendhood.

Three rules should be followed to face the problematics of doctor-patient encounter and the labels that might arise ${ }^{10}$ : the awareness rule - health professionals need to be aware that they are part of a broader social context and that patients bring with them multiple social factors; the avoidance rule - health professionals should avoid reinforcing disadvantages that the patient has already to face; the assumptive rule - health professionals should not assume that difficult patients are the result of one dimension context. So, they should assume that what they bring to the interaction is as important in determining how patients come to be perceived as is what both patients and health professionals actually do or say.

${ }^{9}$ Miranda Fricker. 2007. Epistemic Injustice: Power \& the Ethics of Knowing. London: O.U.P.

10 R. Jones, N. Britten., L. Culpepper, D.A..Gass, D. Mant, R.Grol, C. Silagy, (Eds). (2005). Oxford Textbook of Primary Medical Care. O.U.P. 
As Teresa Casal reminds us ${ }^{11}$ "it takes a dcotor to pronounce you a patient. It takes a person to acknowledge a person. For the patient to be treated as a person in a therapeutic relation, it takes a carer who is aware of his or her cognitive and emotional power and responsibility." Being aware of one's power and responsibility means to acknowledge the person in oneself and our common vulnerability. Wisdom is the main assumption of Narrative Medicine, whether we are referring to educational and training programs, everyday work or scientific research in the healthcare area. Providing physicians with strategies not only for coping with and disclosing their vulnerabilities, but also for facilitating their development of reflective practice could build the path towards wisdom and personal growth. Building a narrative-friendly and narrative-knowledgeable environment could set up a network of Witnesses who would pass on the wisdom and insight gained from their error or near-miss experience, as a "win-win" opportunity for the health care organization, the physician, the patients and their relatives. Finding a place for human errors, and for self-forgiveness, remains challenging within the dominant culture in medical schools, health care organizations and society in general. Human factor is the basis of medical errors, which are multifactorial, deriving from the mental processes of each individual: defects of perception, reasoning, planning, interpretation; failures in teamwork and communication; and failures in the design and articulation of health systems and / or procedures. It is important to give space to the discussion about the error in health care, removing from this discussion the weight of punishment and placing the focus on the relation between error and truth; error and learning; error and burnout; patient error and safety. In addition to systemic approach and error prevention, NM postulates the need for integrating the individual and relational dimensions within the system: error reports do not solve the ethical and moral issues that the error implies on individual, interpersonal, intrateam and inter-team level.

Based on the above mentioned three Rs of narrative-oriented health care (Relation, Recognition of Vulnerability and Reflection), we underline the role of context in analyzing the error committed, as well as the importance of interpretation and perspective: error as technical failure (medical culture); as risk management (hospital administration); as moral failure, injustice, even sin (patient and relatives); as moral stress (doctors, other health professionals). Considering that the main causes of moral distress are communication gaps, collaboration failure among different teams and among members of the same team and the feeling of powerlessness, we can state that, as far as health care errors are concerned, we can only avoid moral residues if we promote communication and strategies for intra-team and inter-team collaboration, and a friendly environment to individual initiative in changing the system.

\section{Reconceptualization as a NM task: Health, body and pain}

Reconceptualizing ourselves as persons and not only as professionals also requires reconceptualizing terms, such as health, body and pain. From a narrative medicine approach, health is not only what a body has, but how a body relates to other bodies and to the environment, being thus perceived as a dynamic concept. Moreover, the body is both the lived body and the object body, which means that the body itself, which is the focus of interest for Evidence-based medicine is always both subjective and objective in terms of its relation to the world and in terms of its place in the world: the body is not only in space but of space and the temporal structure of bodily experience always entangles past and present.

11 Teresa Casal. "Between Patients and Doctors: It Takes a Person". Beyond Diagnosis: Relating the Person to the Patient - The Patient to the Person. Peter Bray and Teresa Casal (Eds.). Oxford: Inter-Disciplinary Press. 2014. p. 102. 
Likewise, pain must be reconceptualized, bearing in mind that how we think about pain is ideally guided by those unspoken and unconscious assumptions, myths, and metaphors that shape our understanding of the individual sufferer's reality and experience of pain. Commonlyutilized diagnostic tests cannot communicate the intensity, duration, quality, and personal dimensions of pain, as this is something that only the sufferer can appreciate. Ideally, physicians observe, listen to, talk with, and trust the patient's narrative of pain, which are skills that physicians are not necessarily taught in their formal training. The experiential gap between the bearer of pain and the observer is so wide that it is said to have an epistemic impact. The believability of pain is confounded by its subjectivity, as is often the case with subjective experience. Pain cannot be generalized on the basis of objective factors that signal a particular therapeutic response. Each individual experiences pain quite differently, depending on factors as variable as neurobiology, culture, religious beliefs, previous experiences of pain, and current psychological and spiritual states of feeling. Narrative medicine stands out as a logical pathway for integration in pain management, as it aims to address each individual patient's experience as a source of data and a resource through which to better attend and understand the pain condition.

Bearing in mind all these considerations on the need for reflection, reconfiguration and reconceptualization,, Narrative Medicine main tasks these days should be to promote training and to address the gap between narrative-based evidence and science-based evidence. Three questions arise within narrative-oriented healthcare:

- How should hospitals be designed and managed, so that they can integrate NM and Evidence-Based Medicine?

- How can technology be integrated into a NM approach, in order to promote high-value care?

- How can narrative skills be implemented and used on a regular basis as part of the clinical reasoning?

In order to preserve the integrity and the humanity of patients and healthcare professionals, these questions have to be addressed by all those who care for a narrative future in healthcare, willing to see with new eyes what has always been there and see it again for the first time:

We shall not cease from exploration

And the end of our exploring

Will be to arrive where we started

And to know the place for the first time

\section{T.S. Eliot, Four Quartets}

\section{References}

Charon. R. (2001). “Narrative Medicine: Form, Function, and Ethics”. Ann Intern Med.;134, pp. 83-87. doi:10.7326/0003-4819-134-1-200101020-00024.

Hurtwitz, B. "Narrative (in) Medicine” (2011). In: Spinozzi P, Hurtwitz B, editores. Discourses and Narrations in the Biosciences. Göttingen: Vandenhoeck \& Ruprecht Unipress, pp.73-87.

Charon,R., DasGupta, S., Hermann, N., Irvine,C., Marcus, E.R., Rivera Colsn, E., Spencer, D., Spiegel, M. (2016). The Principles and Practice of Narrative Medicine . London: O.U.P.

Sackett, DL., Rosenberg, WM., Gray, JA., Haynes RB., Richardson WS. (1996). "Evidence Based Medicine: what it is and what it isn't". Br Med J.,312, pp. 71-2.

Gabbay J. and May AI. (2004) "Evidence based guidelines or collectively constructed "mindlines?" ethnographic study of knowledge management in primary care”. BMJ, 329(7473), p.1013.

Scott, I.A., Soon,J., Elshaug, A.G. and Lindner, R. (2017). “Countering cognitive biases in minimising low value care”. The Medical Journal of Australia. 206 (9), pp. 407-411. doi: 10.5694/mja16.00999 
Murphy, J. W., Min Choi, J., Cadeiras, M. (2016). “The Role of Clinical Records in Narrative Medicine: A Discourse of Message. The Permanente Journal, Spring;20(2), pp. 103-108. http://dx.doi.org/10.7812/TPP/15-101.

Fricker, M. (2007). Epistemic Injustice: Power \& the Ethics of Knowing. London: O.U.P.

Jones, R., Britten, N., Culpepper, L., Gass, D.A., Mant, D., Grol, R., Silagy, C. (Eds). (2005). Oxford Textbook of Primary Medical Care. O.U.P.

Teresa Casal. (2014). "Between Patients and Doctors: It Takes a Person". Beyond Diagnosis: Relating the Person to the Patient - The Patient to the Person. Peter Bray and Teresa Casal (Eds.). Oxford: Inter-Disciplinary Press, pp. 83110. 